\title{
Diagrama de Stacey: um exercício formativo em medicina geral e familiar
}

Filipa Manuel*, Eunice Carrapiço**, Vítor Ramos***

\section{RESUMO}

Enquadramento: O Diagrama de Stacey foi conceptualizado por Ralph Stacey em 1995. Foi desenvolvido como auxiliar no processo de tomada de decisão em situações com graus variáveis de informação, incerteza e complexidade em problemas de gestão e liderança nas organizações. Posteriormente, alguns autores da área da general practice britânica aplicaram este modelo com enfoque especial na abordagem da incerteza e complexidade no processo de decisão clínica. Tomando esta linha conceptual, os autores realizaram o exercício formativo que descrevem neste artigo.

Objetivos formativos: a) refletir criticamente sobre os fatores que influenciam o processo de tomada de decisão em medicina; $b$ ) analisar, com a ajuda do Diagrama de Stacey, os determinantes associados aos problemas de saúde abordados em medicina geral e familiar (MGF); c) discutir, em contexto formativo, as vantagens e limitações do recurso a normas de orientação clínica, guidelines e protocolos; d) reforçar as perceções dos internos quanto à singularidade de cada pessoa e à exigência de uma atitude reflexiva e crítica na abordagem por problemas; e) melhorar a autoconsciência quanto à influência das características pessoais do médico neste processo. Descrição: $O$ exercício consistiu na análise, num dos estágios de MGF, do conteúdo de 30 consultas sequenciais numa semana. $O$ interno, após análise de cada situação, posicionou no Diagrama de Stacey cada um dos problemas abordados, de acordo com as suas perceções e conhecimentos sobre a informação disponível e o consenso atual quanto às intervenções recomendadas. Os resultados foram discutidos no grupo de formação.

Discussão: A mancha gráfica obtida no diagrama, após posicionamento dos problemas segundo a perceção do interno, ilustrou grande diversidade, heterogeneidade e amplitude de variação de graus de incerteza e de consenso associados aos problemas abordados. Cerca de metade dos problemas foram colocados nas zonas de maior complexidade. Apenas um quarto foi colocado na zona que pressupõe maior certeza e maior consenso. Na discussão, o grupo formativo identificou as vantagens do exercício, designadamente na ajuda à perceção da incerteza e da complexidade clínica, pessoal e circunstancial de algumas situações, sobressaindo o caráter dinâmico e intersubjetivo dos processos decisionais em contexto clínico. A replicação do exercício pelos internos de MGF pode aumentar a sua autoconsciência quanto ao processo de raciocínio clínico e reforçar a perceção da influência dos determinantes pessoais e circunstanciais na abordagem de cada problema ou associações de problemas de saúde. Os autores sugerem que outros grupos de formação realizem e discutam entre si os resultados de exercícios semelhantes, como método pedagógico na abordagem da incerteza e da complexidade em medicina, em especial na amplitude clínica da MGF.

Palavras-chave: Formação em Medicina Geral e Familiar; Incerteza; Complexidade; Medicina Geral e Familiar; Diagrama de Stacey.

\section{ENQUADRAMENTO TEÓRICO}

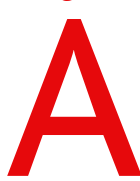

Diagrama de Stacey foi conceptualizado por Ralph Stacey em 1995. Foi desenvolvido como auxiliar no processo de tomada de decisão perante situações com graus variáveis de informação e incerteza na gestão e liderança de organizações. Stacey tem vindo a desenvolver o seu modelo conceptual e interventivo. ${ }^{1-3}$ Alguns autores da área da medicina familiar britânica (general practice) ensaiaram este modelo de análise na sua área médica, com enfoque especial em consultas mais complexas. ${ }^{4-6}$ To- mando esta linha conceptual, os autores realizaram o exercício formativo que descrevem neste artigo.

No Diagrama (Figura 1), o eixo dos X representa o nível decrescente de informação e certeza percecionado pelo decisor sobre um problema, situação e pessoa, in-

* Médica Interna de Medicina Geral e Familiar. USF São João do Estoril, ACES de Cascais

**Médica Especialista de Medicina Geral e Familiar. USF São João do Estoril, ACES de Cascais

***Médico Especialista de Medicina Geral e Familiar. USF São João do Estoril, ACES de Cascais. Professor convidado da Escola Nacional de Saúde Pública (UNL) 
cluindo o conhecimento científico disponível sobre causalidade e determinantes para abordar e compreender um dado problema. O eixo dosY representa, também por ordem decrescente, o grau de evidência ou consenso sobre como atuar em relação a esse problema, desde situações de grande consenso quanto à ação adequada até situações de grande controvérsia quanto ao que fazer. ${ }^{1-3}$

Stacey considerou originariamente no seu Diagrama várias áreas e zonas (Figura 1): ${ }^{-3}$

Zona 1. Mais propensa à «decisão técnica normalizada», por existirem maior certeza e maior consenso e, previsivelmente, maior evidência sobre eficácia e eficiência. Aqui poderão localizar-se as situações

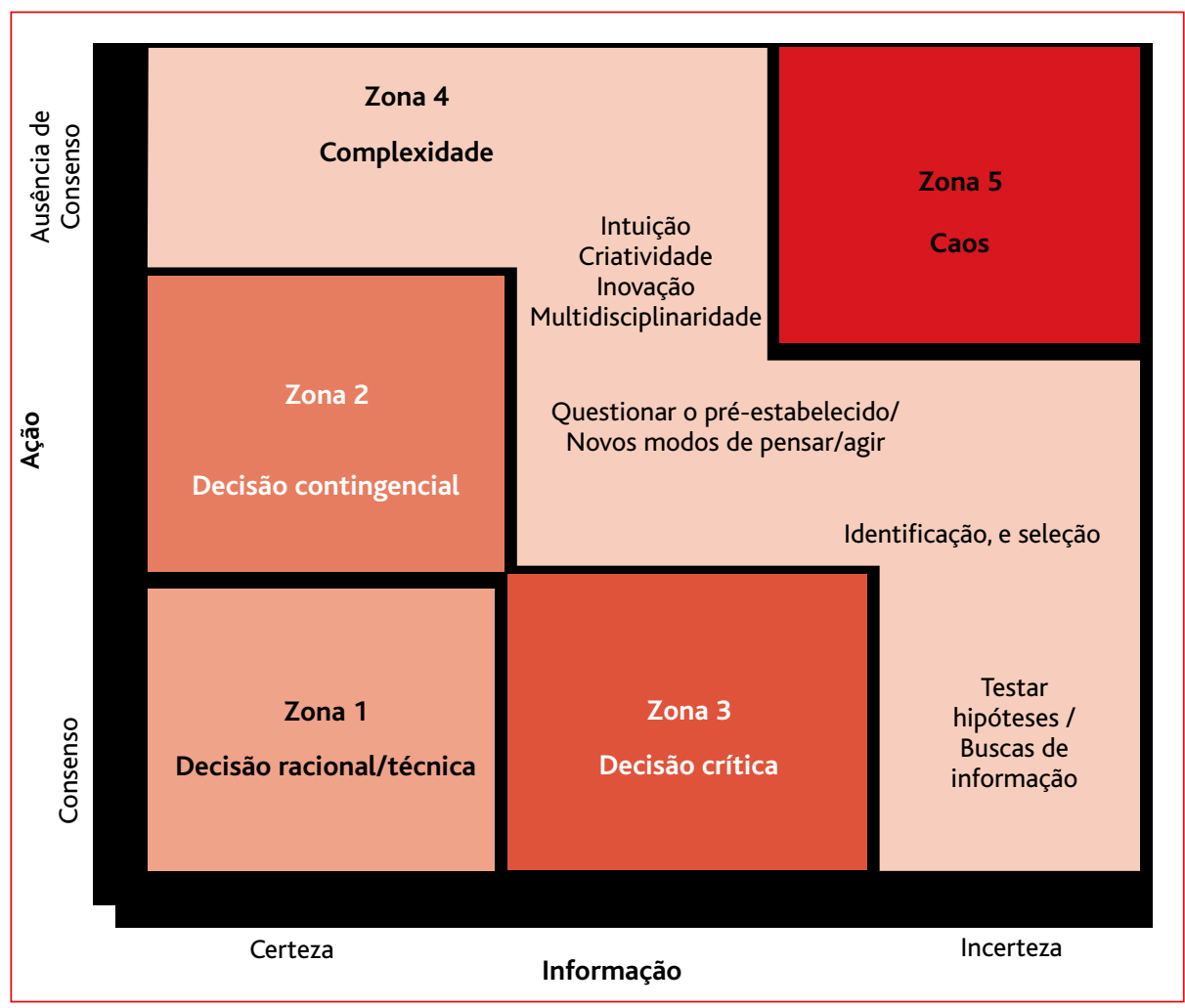

Figura 1. Diagrama de Stacey utilizada no exercício (adaptado de Ramos). ${ }^{7}$ e problemas onde é mais fácil aplicar normas de orientação clínica (NOC), a medicina baseada na evidência (MBE), guidelines e protocolos. ${ }^{4-6}$

Zona2. Suscita «decisões mais contingenciais» por, apesar do grau de informação ou evidência científica disponíveis, existir desacordo quanto ao que se deve fazer. ${ }^{4-6}$

Zona 3. Onde se requerem «decisões mais críticas» por, apesar dos níveis de consenso quanto ao que fazer, existir menor informação ou certeza quanto a mecanismos de causalidade ou caracterização de situação. ${ }^{4-6}$

Zona 4. Zona de maior "complexidade» pela interferência de múltiplos fatores e imprevisibilidade de efeitos e escassez de «receitas feitas». Situa-se entre as zonas 1, 2, 3 e a zona de maior incerteza e controvérsias (zona 5). Aqui requere-se atenção mais abrangente, criatividade e inovação. Nesta zona, as NOC ou guidelines têm utilidade fraca ou nula e, quando consideradas em algum aspeto, devem ser vistas em função de cada pessoa, situação, momento e atendendo à relação médico-doente já construída. ${ }^{4-6}$

Zona 5. Onde existe elevada incerteza e desacordo. Nela pode haver desorganização e contradição de per-

ceções e ações com maior risco de consequências negativas e danos. ${ }^{4-6}$

A área percecionada como de maior segurança (zonas 1,2 e 3) corresponde a menor incerteza e a maior previsibilidade. Na área de maior complexidade (zona 4) crescem a incerteza e a imprevisibilidade, requerendo integração circunstancial de múltiplas variáveis e confronto crítico mais cuidadoso e prudente entre o conhecimento disponível, a realidade de cada pessoa e os seus contextos «aqui e agora». A área de maior risco de desintegração e caos (zona 5) é crítica pela grande instabilidade, insegurança e excessiva incerteza com riscos iminentes e imprevisíveis para a saúde e segurança do doente. Habitualmente requer atenção apurada, discussão interpares, elevada prudência, recursos e ações excecionais.

A translação da conceptualização de Stacey das áreas socio-organizacionais para a medicina e seus processos decisionais é apelativa e intuitiva, tendo sido ensaiada por vários autores, em especial na área da medicina geral e familiar (MGF). ${ }^{4-8}$ Porém, deve ser aplicada com autoconsciência e sentido crítico para evitar o seu uso 
como instrumento simplificador inapropriado. Com a devida cautela, o Diagrama pode ser útil numa reflexão estruturada sobre aspetos e fatores em jogo no raciocínio de decisão face a um problema concreto, mas não uma chave para a sua solução. Esta perspetiva foi adquirida pelos autores no decurso de correspondência trocada com Ralph Stacey, em outubro de 2015.

O contacto com Ralph Stacey permitiu conhecer a sua visão atual de como lidar com a incerteza e a complexidade. Stacey advertiu os autores para alguns inconvenientes do uso acrítico do seu Diagrama, simplificando inadequadamente os processos decisionais na tentativa ilusória de contornar desafios e dificuldades face a situações marcadas pela incerteza, pela mudança e evolução incessantes e pela imprevisibilidade determinada pela contínua emergência de fatores e influências impossíveis de controlar - numa palavra, pela complexidade da vida e, portanto, da medicina. Consequentemente, o Diagrama de Stacey passou a ser visto pelos autores como um auxiliar da tomada de consciência dos níveis de certeza/incerteza e graus de consenso quanto ao que fazer face a determinado problema, numa pessoa singular, mas não como um instrumento para gerar respostas ou soluções.

A sua aplicação consistiu em representar, num dado momento o posicionamento de um problema de saúde apresentado por uma pessoa num dado contexto e momento, admitindo a sua evolução posicional em função da variação de vários fatores, inerentes ao médico, à pessoa e à evolução natural do problema ou doença (Figura 1). Em jogo estão sempre a interação e integração de múltiplos aspetos e fatores, como:

1. O médico cuida de pessoas, o que implica ter em conta vários sistemas interrelacionados (Figura 2): individual, familiar, organizacional, sistema comunitário; ${ }^{8}$

2. Cada problema pode apresentar-se de forma indiferenciada, aguda ou com evolução prolongada, com vários determinantes associados, por vezes não relacionáveis com doença orgânica identificável; 8,10

3. Frequentemente coexistem vários problemas na mesma pessoa com os mais diversos graus de mecanismos de interligação e efeitos emergentes;

4. Podem estar envolvidos comportamentos e estilos de vida, cuja génese e modificação são muito complexas; ${ }^{8,11}$

5. A adesão terapêutica implica a participação e o envolvimento ativo da própria pessoa. Por vezes, o armamentarium terapêutico disponível é insuficiente para determinados problemas, em particular os associados a elevado sofrimento, tendo muitas vezes na sua origem teias familiares, relacionais, laborais, sociais e económicas; ${ }^{8}$

6. A aplicação de tecnologia e a fragmentação disciplinar aumentaram a complexidade das situações

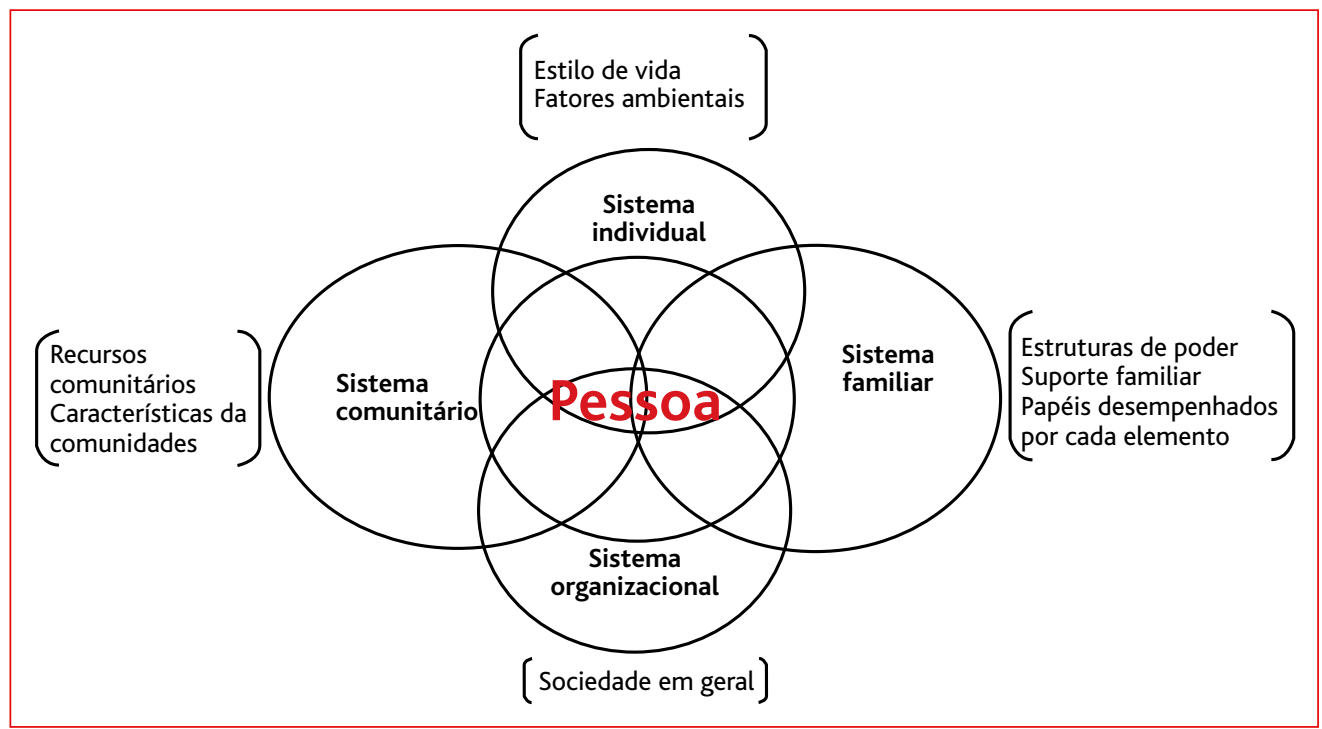

Figura 2. Sistemas interdependentes a ter em conta em cada pessoa. 
como um todo. ${ }^{12}$

Em suma, inerente ao processo de decisão clínica encontra-se uma teia de fatores e aspetos determinantes relacionados com a pessoa, o médico, a circunstância e o problema de saúde da pessoa. Estes fatores despertam incerteza e motivam a mobilização de recursos (e.g., discussão interpares, envolvimento da família e comunidade, referenciação, alargamento do tempo de consulta, etc.). De uma forma simplista contribuem, também, para realçar a complexidade que pode surgir em consultas, o que condiciona uma visão global, uma abordagem holística, centrada na pessoa e na sua circunstância.

O Diagrama de Stacey pode ser utilizado como um auxiliar neste processo de reflexão e análise face a um problema de saúde. Contudo, o modo de o ver e de analisar deve ter em conta o todo da pessoa, a globalidade da sua situação clínica e dos seus contextos e circunstâncias. Não é possível posicionar uma pessoa num Diagrama bidimensional e estático, apenas nos é possível posicionar a pessoa com um dado problema, num dado momento, integrada na sua rede de sistemas e em interação com um determinado médico. ${ }^{1-3}$

\section{DESCRIÇÃO DO EXERCÍCIO}

O exercício foi realizado durante um estágio de MGF do internato da especialidade, no âmbito de um dos objetivos educacionais indicados pela coordenação do internato. Os seus objetivos formativos específicos foram:

a) Refletir criticamente sobre os fatores que influenciam o processo de tomada de decisão em medicina;

b) Analisar, com a ajuda do Diagrama, determinantes como: nível de informação; previsibilidade evolutiva e incerteza associadas ao diagnóstico, prognóstico, ações e resultados associados aos problemas de saúde abordados em MGF;

c) Discutir em contexto formativo as vantagens e limitações do recurso a normas de orientação clínica, guidelines e protocolos;

d) Reforçar as perceções dos internos quanto à singularidade de cada pessoa e à exigência de uma atitude reflexiva e crítica na abordagem por problemas;

e) Melhorar a autoconsciência quanto à influência das características pessoais do médico neste processo.

$\mathrm{O}(\mathrm{s})$ potencial(ais) ganho(s) com a aplicação do exercício, na perspetiva da formação do interno são:

a) Treino de um raciocínio reflexivo sobre os fatores influenciadores do processo de tomada de decisão numa consulta; 


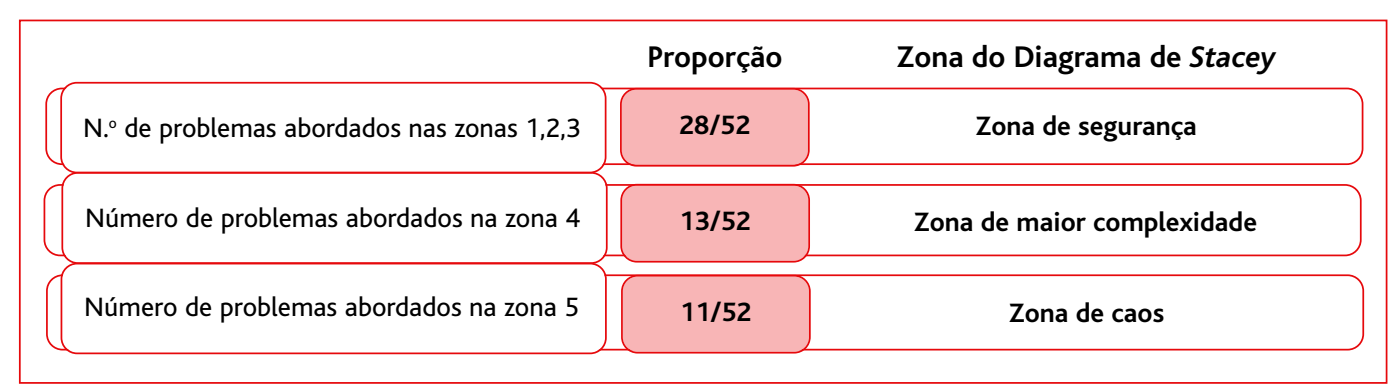

Figura 4. Distribuição pelas zonas do Diagrama de Stacey, dos problemas abordados em 30 consultas a pessoas diferentes durante uma semana.

b) Identificação de necessidades pessoais de aprendizagem;

c) Utilização do diagrama como instrumento auxiliador do processo reflexivo e formativo de um interno ou médico especialista.

O exercício visou, em particular, aprofundar os temas da subjetividade/objetividade na decisão clínica, bem como os da diversidade, abrangência, incerteza e complexidade em MGF. Este exercício serviu exclusivamente fins de aprendizagem e treino e não deve ser visto nem interpretado como um estudo.

A metodologia do exercício consistiu na análise do conteúdo de consultas de MGF no período de uma semana, que corresponderam a 30 consultas consecutivas, e posicionar cada um dos problemas abordados num ponto do Diagrama de Stacey, de acordo com as perceções e conhecimentos do interno sobre a informação disponível e consenso atual quanto às intervenções recomendadas. Os critérios para posicionamento, tendo em conta os eixos do X e do Y, foram os determinados pela perceção subjetiva do interno. Os resultados foram discutidos em grupo de formação e apresentados em reunião de equipa da unidade de saúde. Foram posicionados no diagrama 52 problemas (Figura 3, Quadro 1). Estes números decorrem da realização de consultas consecutivas para o período de tempo estipulado para o exercício e incluem consultas de saúde do adulto, saúde infantil, planeamento familiar e saúde da mulher e consulta não programada.

\section{DISCUSSÃO}

A representação obtida no Diagrama de Stacey representa uma «mancha» onde cerca de metade dos problemas foi posicionada nas áreas de maior segurança (zonas 1, 2 e 3). A restante metade dos problemas foi posicionada em zonas com maior incerteza e imprevisibilidade. Cerca de um quinto dos problemas foram posicionados na área mais crítica que Stacey designa no seu diagrama como zona de «caos» (Figura 3).

Salienta-se o facto de cerca de metade dos problemas abordados terem sido percecionados fora dos quadrantes onde é possível e mais fácil aplicar conhecimentos com maior evidência científica, designadamente os disponíveis em NOC e guidelines. A abordagem contextualizada destes problemas é um dos grandes desafios da prática da MGF. Apela a uma maior prudência, a análises, consultorias e discussão interpares, ao envolvimento multidisciplinar, a atitudes de permanente questionamento crítico e a uma abordagem sempre centrada na pessoa e nas suas circunstâncias. Em suma: maior conjugação e integração de conhecimentos, com envolvimento de outros recursos, profissionais e disciplinas. ${ }^{13}$ De igual modo, surge como indispensável o envolvimento e a participação dos doentes para encontrar soluções satisfatórias.

A análise qualitativa detalhada de cada situação permite ao interno refletir e questionar sobre diversos ângulos o seu processo de análise e decisão clínica, como: o mesmo problema em pessoas, situações e momentos diferentes pode ser percecionado em diferentes zonas do diagrama. Por exemplo:

1. $\mathrm{O}$ «excesso de peso» tanto pode ser posicionado na zona 1 se se tratar do motivo de consulta e reconhecido como um problema pelo(a) doente ou na zona 4 quando não é relevante para a pessoa e requer uma forma personalizada, cautelosa e indireta de o abordar;

2. A «surdez» pode cair na zona 5 quando, apesar de 
parecer que a consulta correu bem, com feedback positivo por parte da utente, no fim da mesma, a pessoa não é capaz de reproduzir a informação veiculada. Esta situação exemplifica um desencontro entre o desempenho do médico e o efeito obtido. Mas a «surdez» pode também ser posicionada noutras zonas, requerendo abordagens diversas e a busca de soluções com o envolvimento da pessoa e outros recursos como foi o caso de uma puérpera surda, a habitar com mãe alcoólica e com receio de não prestar os melhores cuidados ao filho devido à sua deficiência;

3. A «hipertensão arterial» tanto pode ser posicionada na zona 1 quando corresponde a hipertensão arterial essencial, bem controlada, ou ser colocada numa das zonas de maior complexidade noutra pessoa com problemas económicos graves, sem possibilidade de comprar os fármacos anti-hipertensores.

Portanto, muito para além dos problemas ou entidades nosológicas em si, são determinantes as características de cada pessoa e dos seus contextos, circunstâncias e momento, bem como as perceções, os conhecimentos e a experiência de cada médico.

As guidelines e as NOC são desenhadas para situações ou doenças específicas, excluindo frequentemente as fases extremas da vida e as situações de multimorbilidade, cada vez mais frequentes. Também não atendem aos contextos, à singularidade e ao momento de cada pessoa. Por isso, sendo úteis, devem ser usadas sempre com uma atitude de questionamento crítico. ${ }^{14}$

Por outro lado, é nas zonas de maior incerteza e imprevisibilidade que a medicina sobressai como «arte», que complementa a ciência e que questiona os conhecimentos existentes ou inexistentes, estimulando a sua evolução. É também por esta via que podem surgir novas perguntas de investigação, estimulando o ensaio de novos «modos de ver» e de «fazer diferente» e se produzem novos conhecimentos.

A aplicação do Diagrama de Stacey numa se-

\begin{tabular}{|c|c|c|}
\hline \multicolumn{3}{|c|}{$\begin{array}{l}\text { QUADRO I. Chave da numeração atribuída pelo médico aos } \\
\text { problemas abordados, segundo a classificação ICPC-2 (Figura 3) }\end{array}$} \\
\hline $\begin{array}{l}\text { Número } \\
\text { atribuído }\end{array}$ & $\begin{array}{l}\text { Código } \\
\text { ICPC-2 }\end{array}$ & Designação ICPC-2 \\
\hline 1 & D93 & Síndroma do cólon irritável \\
\hline 2 & P20 & Alterações da memória \\
\hline 3 & K86 & Hipertensão sem complicações \\
\hline 4 & D26 & Medo de cancro do aparelho digestivo \\
\hline 5 & T90 & Diabetes tipo 2 \\
\hline 6 & T90 & Diabetes tipo 2 \\
\hline 7 & T93 & Alterações do metabolismo dos lípidos \\
\hline 8 & $\mathrm{X} 72$ & Candidíase genital na mulher \\
\hline 9 & $\mathrm{Z} 22$ & Problema por doença de familiar \\
\hline 10 & T93 & Alterações do metabolismo dos lípidos \\
\hline 11 & K76 & Doença cardíaca isquémica sem angina \\
\hline 12 & K86 & Hipertensão sem complicações \\
\hline 13 & Y85 & Hipertrofia prostática benigna \\
\hline 14 & L86 & Síndroma vertebral com irradiação de dores \\
\hline 15 & L91 & Outras osteoartroses - rizartrose \\
\hline 16 & D97 & Doença do fígado - hepatite B \\
\hline 17 & $\mathrm{X} 11$ & Sintomas da menopausa - sintomas vasomotores \\
\hline 18 & K86 & Hipertensão sem complicações \\
\hline 19 & L90 & Osteoartrose do joelho \\
\hline 20 & A92 & Alergia/reacção alérgica \\
\hline 21 & P01 & Sensação ansiedade/nervosismo/tensão \\
\hline 22 & N87 & Parkinsonismo \\
\hline 23 & P76 & Perturbações depressivas \\
\hline 24 & T90 & Diabetes tipo 2 \\
\hline 25 & K78 & Fibrilhação auricular \\
\hline 26 & K87 & Hipertensão com complicações \\
\hline 27 & N01 & Cefaleia \\
\hline 28 & L83 & Síndroma da coluna cervical \\
\hline 29 & L18 & Dor muscular - lombalgia aguda \\
\hline 30 & P74 & Distúrbio ansioso/estado de ansiedade \\
\hline 31 & Y99 & Doença genital masculina - ejaculação dolorosa \\
\hline 32 & K86 & Hipertensão sem complicações \\
\hline 33 & A92 & Alergia/reacção alérgica \\
\hline 34 & R74 & Infecção aguda do aparelho respiratório superior \\
\hline 35 & K96 & Hemorróidas \\
\hline
\end{tabular}




\begin{tabular}{|c|c|c|}
\hline \multicolumn{3}{|c|}{ QUADRO I. (continuação) } \\
\hline $\begin{array}{l}\text { Número } \\
\text { atribuído }\end{array}$ & $\begin{array}{l}\text { Código } \\
\text { ICPC-2 }\end{array}$ & Designação ICPC-2 \\
\hline 36 & P74 & Distúrbio ansioso/estado de ansiedade \\
\hline 37 & Z21 & Problema comportamental de familiar \\
\hline 38 & Z05 & Problemas com as condições de trabalho \\
\hline 39 & R76 & Amigdalite aguda \\
\hline 40 & U71 & Cistite/outra infecção urinária \\
\hline 41 & L95 & Osteoporose \\
\hline 42 & K86 & Hipertensão sem complicações \\
\hline 43 & N89 & Enxaqueca \\
\hline 44 & L90 & Osteoartrose do joelho \\
\hline 45 & X89 & Síndrome de tensão pré-menstrual \\
\hline 46 & R96 & Asma \\
\hline 47 & W15 & Infertilidade \\
\hline 48 & A04 & Debilidade/cansaço geral - a esclarecer \\
\hline 49 & B80 & Anemia por deficiência de ferro \\
\hline 50 & P76 & Perturbações depressivas \\
\hline 51 & T83 & Excesso de peso \\
\hline 52 & W82 & Aborto espontâneo \\
\hline
\end{tabular}

Nota: O posicionamento do problema na matriz dependeu de múltiplos factores relacionados com a pessoa, os seus contexto, a natureza e particularidades do problema, a comorbilidade, a polifarmacoterapia e as perceções e conhecimentos e experiência do médico.

mana de consultas e a sua discussão num grupo de formação permitiu evidenciar características, dilemas e exigências da prática clínica da MGF, designadamente:

- A amplitude da dispersão quanto à incerteza e imprevisibilidade associadas aos problemas que um médico pode abordar num limitado período de tempo;

- O posicionamento de um dado problema na matriz depende da pessoa, das suas circunstâncias e momento, das perceções, conhecimentos e exigências do médico e, também, da natureza e estádio do problema na sua história evolutiva natural,

- Cerca de metade dos problemas abordados foram percecionados, embora com dispersão, numa zona de maior certeza e maior segurança para o clínico;

- Em contrapartida, os restantes problemas (também cerca de metade), por fatores pessoais, pela sua natureza, estádio evolutivo, comorbilidades associadas, polifarmacoterapia e outros fatores, foram percecionados e posicionados na matriz em zonas de maior incerteza e complexidade;

- Os autores recomendam a utilização do exercício tendo por base uma visão alargada, considerando cada pessoa e a sua constelação de problemas;

- Dadas as limitações da mente humana, mesmo tendo a preocupação de uma abordagem holística centrada na pessoa, a operacionalização do raciocínio clínico com aplicação do conhecimento científico disponível remete inexoravelmente para uma fase analítica por problemas, ainda que possa e deva passar a etapas mentais de integração e tentativas de síntese dos múltiplos fatores e circunstâncias presentes;

- O posicionamento de cada problema no Diagrama depende, também, das perceções, conhecimentos e experiência do médico - processo com uma indissociável mistura de subjetividade/objetividade de que o médico deve estar autoconsciente e ser capaz de um permanente questionamento reflexivo e crítico.

Em conclusão: O Diagrama de Stacey, se usado acriticamente pode ser redutor e escamotear a contigencialidade, a coevolutividade e a incerteza inerentes a cada pessoa, suas circunstâncias, seus problemas de saúde, seus cuidadores e intervenções terapêuticas. Porém, o mesmo Diagrama usado com atitude crítica serviu, neste exercício, como um instrumento facilitador de análises e reflexões formativas como as descritas neste artigo. Por isso, os autores sugerem que outros internos ou médicos especialistas realizem exercícios idênticos, discutam interpares ou em comunidades formativas e comparem os seus resultados e reflexões com os partilhados neste artigo.

\section{REFERÊNCIAS BIBLIOGRÁFICAS}

1. Stacey RD, Griffin D, Shaw P. Complexity and management, fad or radical challenge to system thinking. London: Routledge; 2000. ISBN 0415247616

2. Stacey RD. Complexity and group processes: a radically social understanding of individuals. London: Routledge; 2014. ISBN 9781138011977 
3. Stacey RD. The tools and techniques of leadership and management: meeting the challenge of complexity. London: Routledge; 2012. ISBN 978041553118-4

4. Griffiths $F$, Byrne $D$. General practice and the new science emerging from the theories of 'chaos' and complexity. Br J Gen Pract. 1998;48(435): 1697-9.

5. Innes AD, Campion PD, Griffiths FE. Complex consultations and the 'edge of chaos. Br J Gen Pract. 2005;55(510):47-52.

6. www.gp-training.net - The GP education and training resource [homepage]. Brad Cheek; 2016 Feb [cited 2015 Mar 15]. Available from: http://www.gp-training.net/

7. Ramos V.A consulta em sete passos: execução e análise crítica de consultas em medicina geral e familiar. Lisboa:VFBM Comunicação; 2008. ISBN 9789898160225

8. Tudela M, Lobo FA, Ramos V. Desafios da complexidade em medicina geral e familiar [The challenges of complexity in family medicine]. Rev Port Clin Geral. 2007;23(6):715-25. Portuguese

9. Stewart M. Patient-centered medicine: transforming the clinical method. 2nd ed. Abingdon: Radcliffe Medical Press; 2003. ISBN 9781857759815

10. Nunes JM. Sintomas somatoformes em medicina geral e familiar [Dissertation]. Lisboa: Faculdade de Ciências Médicas da Universidade Nova de Lisboa; 2012.Available from: http://run.unl.pt/handle/10362/10527 11. Miller WR, Rollnick S. Motivational interviewing: helping people change. 3rd ed. New York: Guilford Press; 2013. ISBN 9781609182274

12. Martin JC. O direito do doente à informação: contextos, práticas, satisfação e ganhos em saúde [Dissertation]. Porto: Instituto de Ciências Biomédicas Abel Salazar da Universidade do Porto; 2008. Available from: http://repositorio-aberto.up.pt/handle/10216/7251

13. Nicolescu B. O manifesto da transdisciplinaridade. São Paulo: TRIOM; 1999.

14. Greenhalgh T, Howick J, Maskrey N. Evidence based medicine: a movement in crisis? BMJ. 2014;348:g3725.

\section{CONFLITO DE INTERESSES}

Os autores declaram não ter conflitos de interesses.

\section{ENDEREÇO PARA CORRESPONDÊNCIA}

Filipa Melo da Silva Manuel

Av. de Roma, n. ${ }^{\circ} 8-2 .^{\circ}$ esq. 1000-265 Lisboa

E-mail: filipa.m.s.m@gmail.com

Recebido em 28-05-2015

Aceite para publicação em 03-04-2016

\section{ABSTRACT}

\section{THE STACEY DIAGRAM: A FORMATIVE EXERCISE IN FAMILY MEDICINE}

Background: Ralph Stacey designed the Stacey diagram in 1995 to help in the analysis of decision-making in situations with different degrees of information, complexity, and uncertainty in management and leadership within organizations. British General Practitioners adapted this model to the medical field, with a focus on more complex and uncertain situations. This article describes a training exercise using the Stacey diagram in a clinical setting.

Training objectives: We attempted to critically reflect on the factors that influence the decision-making process in medicine, to analyse the determinants of health problems in General Practice consultations using the Stacey diagram, to discuss the advantages and limitations arising from the use of guidelines and protocols in a training context, to strengthen the trainees' perceptions of the uniqueness of each person and the need for a reflective and critical attitude to deal with health problems, and to raise awareness of the influence of the personal characteristics of the physician in this process.

Description: The exercise consisted of the analysis of the content of 30 sequential consultations in one week in a training program in general practice. The trainee placed each health problem encountered on the Stacey Diagram based on her perceptions and knowledge of available information about the problem and consensus regarding recommended actions. The results were discussed in the training group. Discussion: The diagram obtained illustrates diversity, heterogeneity, and the range of uncertainty and consensus associated with the health problems addressed. Nearly half of the health problems were placed in areas with higher complexity. Only one quarter of the problems were placed in the area that signifies greater certainty and greater consensus. The training group identified the advantages of the exercise, in particular support for the perception of uncertainty and clinical complexity, based on individual factors in some circumstances. This highlights the dynamic and subjective nature of the decision-making process in medicine. The replication of this exercise by a GP-trainee can increase self-awareness about the clinical decision making process and enhance perception of the influences of personal and situational determinants for each health problem. The authors suggests that other training groups should perform and discuss similar exercises as a training method to approach uncertainty and complexity in medicine in general and in general practice in particular.

Keywords: Family Medicine Training; Uncertainty; Complexity; General Practice; Family Medicine; Stacey Diagram. 